\title{
PROOF OF AN INEQUALITY CONJECTURE FOR A POINT IN THE PLANE OF A TRIANGLE
}

\author{
Yong Yang, Shengli Chen, Dong Huang, \\ Xiang Wang AND XiaOgUANG LiN
}

Abstract. In [1] Jian Liu established a novel inequality about an arbitrary point in the plane of a triangle. He also put forward a conjecture about a parameterized version of this inequality. In this paper, we proceed to give a proof of this inequality facilitated by a combination of computeraided calculations and traditional planar geometry. This proof demonstrates again the strengths of the real algebra methodology develped over time by Ritt, Wu, Yang, Yang, Xia, et. al.

Mathematics subject classification (2010): 51M16, 51N20.

Keywords and phrases: Triangle, point, difference substitution, conjecture.

\section{REFERENCES}

[1] JIAN LIU, A new inequality for a point in the plane of a triangle, Journal of Mathematical Inequalities, 2014, 8 (1): 597-611.

[2] Wenjun Wu, Mathematics Mechanization, Beijing, Science Press, 2003.3.

[3] LU YANG, BICAN XIA, Inequality proving and automatic discovery, Beijing, Science Press, 2008.1.

[4] Shengli Chen, Yong Yao, Jia Xu, The method of split Dimension reduction algebraic inequality and Machine proof, System science and Mathematics, 2009, 29 (1): 26-34.

[5] Shengli Chen, Liangyu Chen, The Degree-Decreasing decomposition of symmetric forms with a program for algebraic inequality decision, System science and Mathematics, 2013, 33 (2): 179-196.

[6] Shengli Chen, FAngJiAn HuAng, Schur decomposition for symmetric ternary forms and readable proof to inequalities, Journal of Mathematics, 2006, 49 (3): 491-502.

[7] F. J. HuAng, S. L. CHEN, Schur partition for symmetric ternary forms and readable proof to inequalites, Proceedings of the 2005 International Symposium on Symbolic and Algebraic Computation, ACM press 2005: 185-192.

[8] L. YANG, X. Hou, Z. ZENG, A complete discrimination symsten for polynomials, China E, 1996, 39 (6): 628-646.

[9] Lu YAng, BicAn XIA, Quantifier Elimination for Quartics, Artificial Intelligence and Symbolic Computation, Lecture Notes in Computer Science, Vol. 4120, (2006), pp. 131-145

[10] Lu YAng, Jingzhong Zhang, Xiaorong Hou, Nonlinear algebraic equations and theorem Machine proving, Shanghai: Shanghai science and Education Press, 1996.9. 